\title{
Nanofluidic Flowmeter Using Carbon Sensing Element
}

\author{
${ }^{1,2}$ Yoshihiro Mizuno, ${ }^{2}$ Matthieu Liger, and ${ }^{2} Y u$-Chong Tai \\ ${ }^{1}$ Fujitsu Laboratories Ltd., \\ 64, Nishiwaki, Ohkubo, Akashi, 674-8555, Japan \\ E-mail: mizuno.yoshi@jp.fujitsu.com \\ ${ }^{2}$ Caltech Micromachining Lab., California Institute of Technology, \\ 1200 E. California Blvd., Pasadena, CA 91125, USA
}

\begin{abstract}
The field of nanofluidics dealing with $\mathrm{nL}$ fluids is growing, and sensors for monitoring ever smaller flow rates $(\sim \mathrm{nL} / \mathrm{min})$ are needed. This paper presents a new, sensitive micromachined thermal sensor for measuring flow rates. The integrated sensor uses a high-TCR (temperature coefficient of resistance) carbon sensing element obtained from ion-implanted parylene. The ion-implanted carbon element has a high temperature coefficient of resistance of $-2 \% /{ }^{\circ} \mathrm{C}$ and is embedded in a freestanding microchannel suspended from the substrate. The developed sensor has been characterized for flow measurements with a volumetric flow sensitivity of $380 \mu \mathrm{V} /(\mathrm{nl} / \mathrm{min})$ under a constant current bias with a power consumption of only $28 \mu \mathrm{W}$. To our knowledge, this is the first such nanofluidic carbon flow sensor and its sensitivity is better than any of flow sensors reported to date.
\end{abstract}

\section{INTRODUCTION}

MEMS and NEMS (Micro- and Nano- ElectroMechanical Systems) are enabling micro- and nano- fluidic systems for many applications, such as dosing systems, chemical microplants, and biomedical detection. Precise flow control is needed in these applications. Conventional commercial sensors are insufficient for developing integrated systems because of their limited sensitivity, large size, and difficulties in integration with other devices.

MEMS and NEMS technologies have been used to develop many types of flow sensors that would be easy to integrate with fluidic systems and enable precise flow control $[1,2]$. To achieve a high enough resolution in the flow measurement, both thermal and non-thermal principles have been used in systems with microchannels fabricated with chip-bonding techniques, surface micromachining techniques (without chip-bonding), PDMS techniques, and parylene surface micromachining techniques. Of these techniques, development of parylene surface micromachining technology that enables the integration of various fluidic devices has been a long-term goal for us [3].

This paper presents a first nanofluidic carbon-ionimplanted-parylene flow sensor for measuring liquid flow rates. The thermal flow sensor uses a carbon ion-implanted parylene register embedded within the wall of a parylene microchannel. The microchannel, a key feature of the sensor, is fabricated using parylene surface micromachining technology and released from the silicon substrate (by using $\mathrm{XeF}_{2}$ gas etching) for improved thermal isolation. The ion-implantation condition is optimally chosen for an increased temperature coefficient of resistance (TCR).

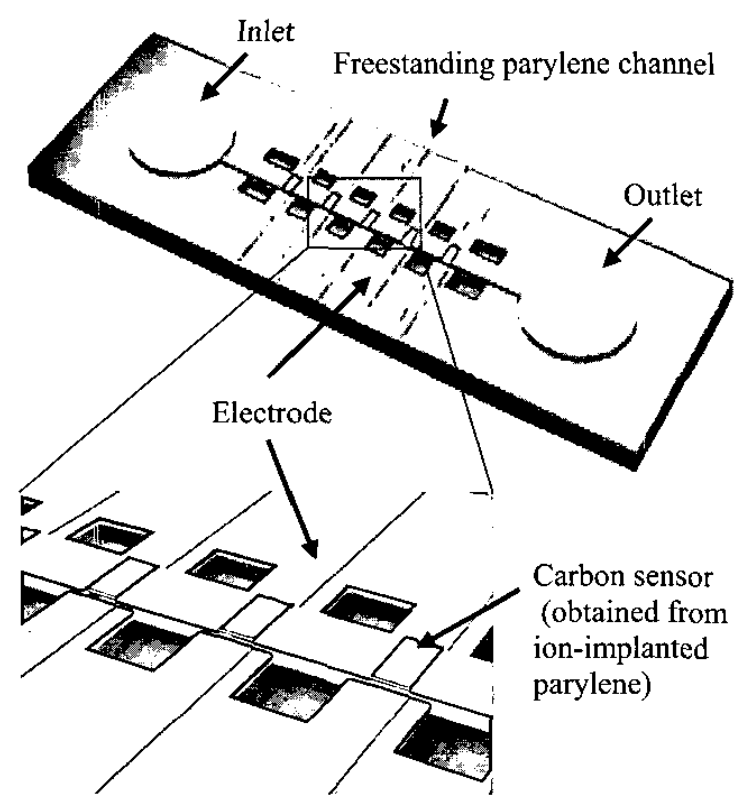

Figure 1: Carbon sensor integrated with freestanding parylene channel.

\section{DEVICE STRUCTURE}

Figure 1 illustrates the concept of the carbon sensor (obtained from ion-implanted parylene) integrated with the freestanding parylene microchannel. The basic operating principle is that the flow sensor utilizes a convective heat transfer from the carbon sensor. The carbon sensor is directly built into the parylene channel wall. The flow in the channel can be detected by the measuring the sensor's temperature. The metal electrodes serve as an electrical conductor for the carbon sensor. The ion implantation condition is optimal for temperature sensitivity and operational and fabrication stability. A portion of the substrate underneath the channel has been removed to increase thermal isolation and the sensor's sensitivity to the fluid flow.

\section{FABRICATION}

The process flow is shown in Figure 2. The devices were fabricated using a two-layer parylene process with ion implantation, photoresist as the sacrificial layer, and gold as the electrode material.

The process started with a 4 -inch wafer with $1.6-\mu \mathrm{m}$ thermal oxide. The oxide was patterned on both sides, and the 


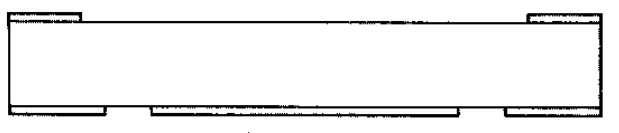

Oxide patterning

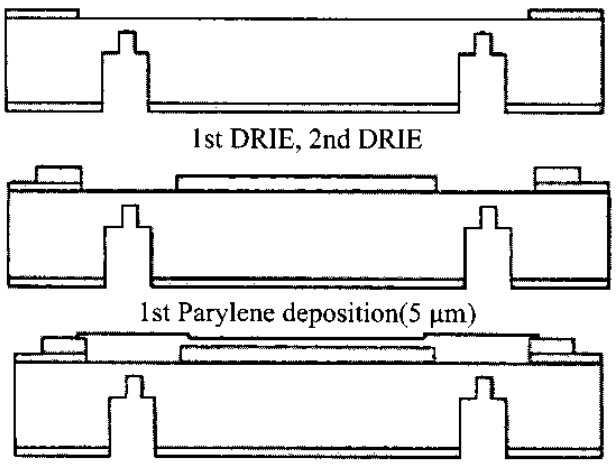

Photoresist for step coverage,

Photoresist for sacrificial layer $(2 \mu \mathrm{m})$

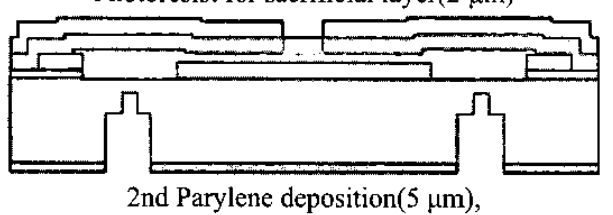

Two-layer photoresist process,

Ion implantation

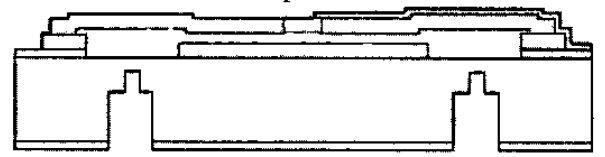

Parylene etching, Metal patterning

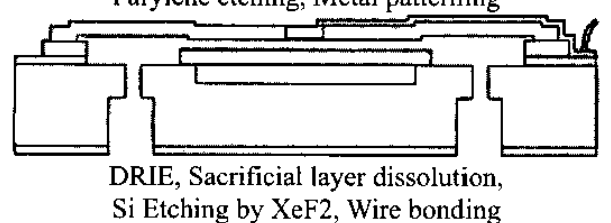

Figure 2: Fabrication process.

back side was DRIE-etched. A 5- $\mu$ m parylene-C deposition was done on the front side after coating with an adhesion promoter. After parylene etching, the step coverage for the $5-\mu \mathrm{m}$ step of the inlet and the outlet was done by using $8-\mu \mathrm{m}$ photoresist. After a $2-\mu \mathrm{m}$ photoresist was patterned for the channel, another $5-\mu \mathrm{m}$ parylene-C deposition was done. Oxygen plasma cleaning and roughening was performed before the parylene deposition to improve the adhesion between the parylene layers. A carbon ion implantation (100 $\mathrm{keV}$ energy and $1.0 \times 10^{16}$ atoms $/ \mathrm{cm}^{2}$ dose) was done with a photoresist mask. A two-layer photoresist mask was used to strip away the carbonized photoresist [4]. First, a thin layer of photoresist was spun onto the wafer and globally exposed. On top of this layer, a second thicker photoresist was spun and patterned. An undercut of the first layer enabled the carbonized photoresist stripping. The completed carbon sensing element is shown in Figure 3. An electrode with a $2000 \AA$ gold layer was formed by using lift-off technology.
Before lift-off, oxygen plasma cleaning and roughening was performed to improve the adhesion between the parylene layer and the gold layers. The two-layer photoresist process was also used to improve the fabrication stability for lift-off The inlet and the outlet were DRIE-etched through the wafer, and the sacrificial photoresist layer was dissolved with acetone and isopropyl alcohol. The freestanding microchannel was completed by etching the Si underneath the channel with $\mathrm{XeF}_{2}$ gas.

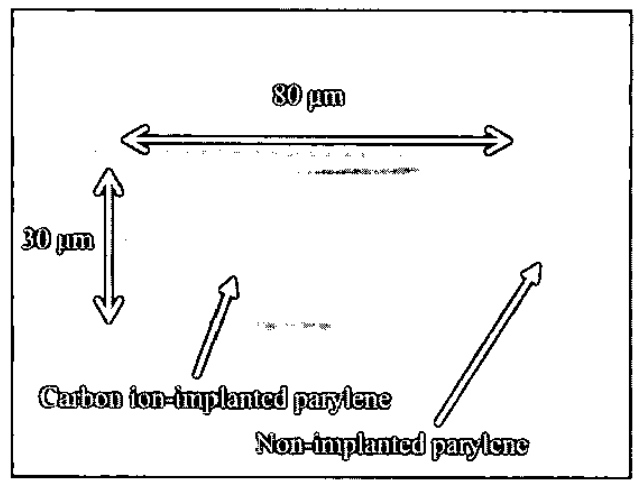

Figure 3: Carbon sensing element.

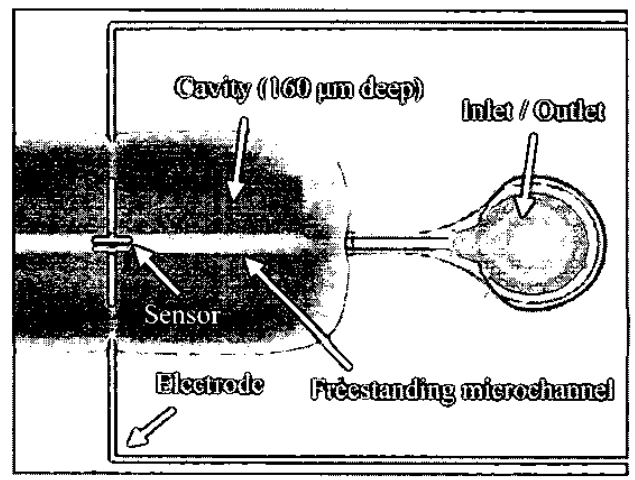

Figure 4: Fabricated carbon sensor with freestanding microchannel.

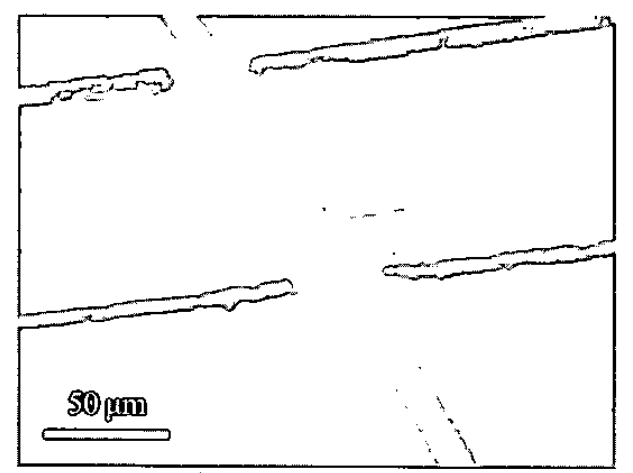

Figure 5: SEM photograph of sensor and freestanding channel. 


\section{RESULTS AND DISCUSSION}

Figure 4 shows a photograph of the carbon sensor with the freestanding microchannel, and Figure 5 shows an SEM photograph. The channel is $20-\mu \mathrm{m}$ wide and $2-\mu \mathrm{m}$ thick. Figure 6 shows a cross-sectional SEM photograph of the sensor and the channel (before $\mathrm{XeF}_{2}$ gas etching). The electrode is placed just on the top of 2nd parylene including the implanted parylene. Figure 7 shows a close-up SEM photograph, and Figure 8 shows a close-up STEM photograph (FIB-prepared cross-sections).

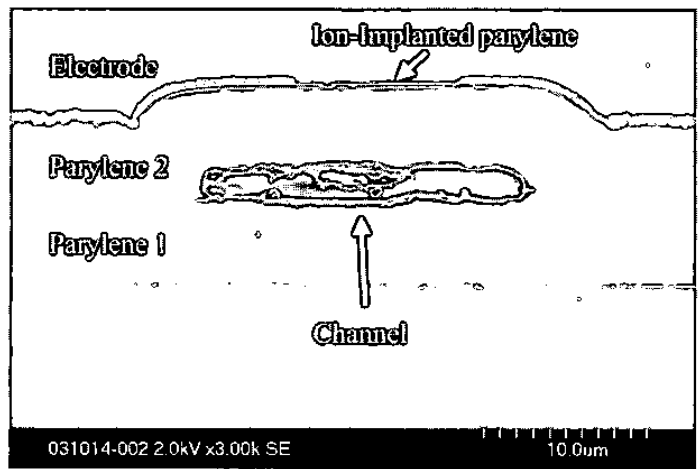

Figure 6: Cross-sectional SEM photograph of channel.

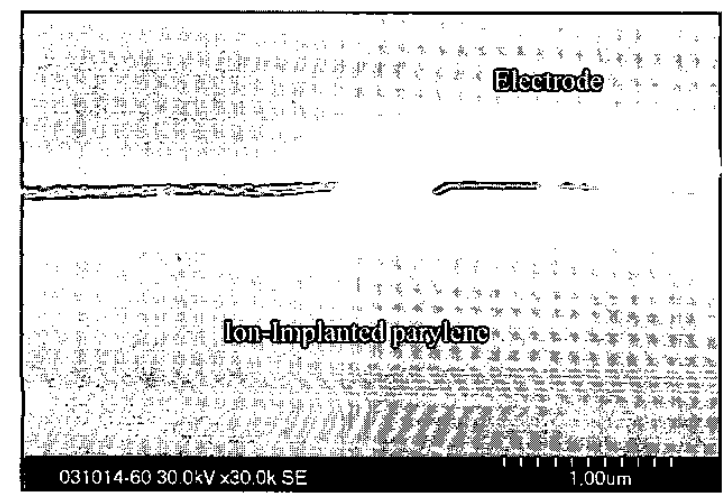

Figure 7: Close-up SEM photograph of carbon sensor.

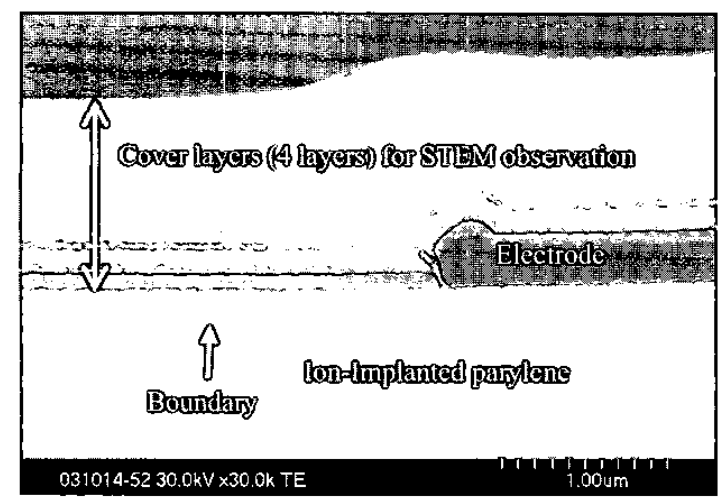

Figure 8: Close-up STEM photograph of carbon sensor.
The implanted layer can be seen in the upper layer of the parylene in both photographs. In particular, the $0.2-\mu \mathrm{m}$-thick layer from the top surface can be distinguished in the STEM photograph. The FTIR spectrum from the non-implanted parylene is shown in Figure 9, and that from the ion-implanted parylene is shown in Figure 10. Two peaks stand out in the FTIR spectrum of the ion-implanted parylene in comparison with the spectrum of the non-implanted parylene. The peak from $3200 \mathrm{~cm}^{-1}$ to $3800 \mathrm{~cm}^{-1}$ is probably due to $\mathrm{O}-\mathrm{H}$ absorption in the implanted parylene. The peak at $1700 \mathrm{~cm}^{-1}$ is probably due to $\mathrm{C}=\mathrm{O}$ absorption in the implanted parylene. These peaks are probably due to an oxidized implanted layer. The Raman spectra from non-implanted (Excitation: $514.5 \mathrm{~nm}, 1.0 \mathrm{~mW}$ ) and implanted parylene (Excitation: $514.5 \mathrm{~nm}, 0.1 \mathrm{~mW}$ ) are shown in Figures 11 and 12 , respectively. The peak at approximately $1600 \mathrm{~cm}^{-1}$ in the implanted parylene is probably due to amorphous carbon. We also confirmed that the etching rate of the carbon layer is lower than that of the non-implanted parylene.

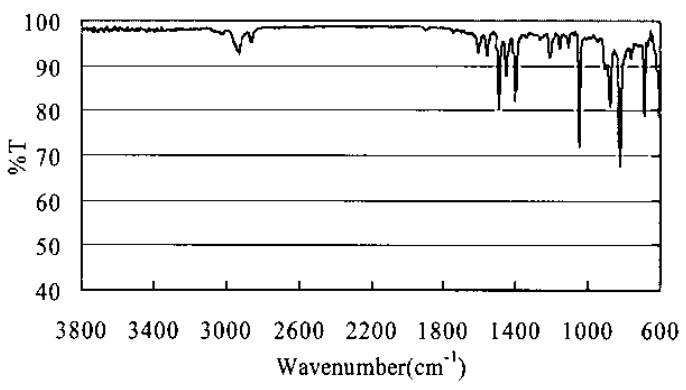

Figure 9: FTIR spectrum from non-implanted parylene.

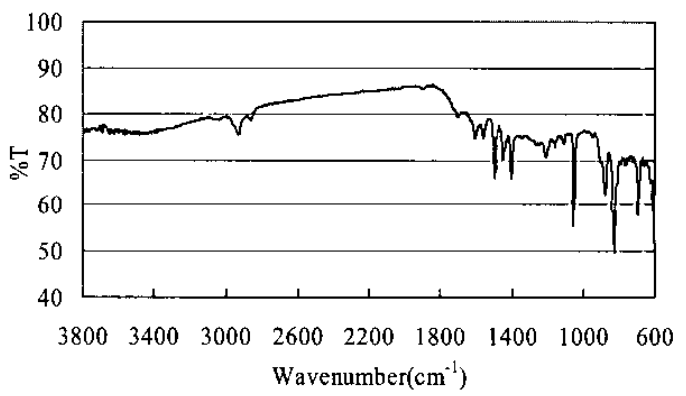

Figure 10: FTIR spectrum from ion-implanted parylene.

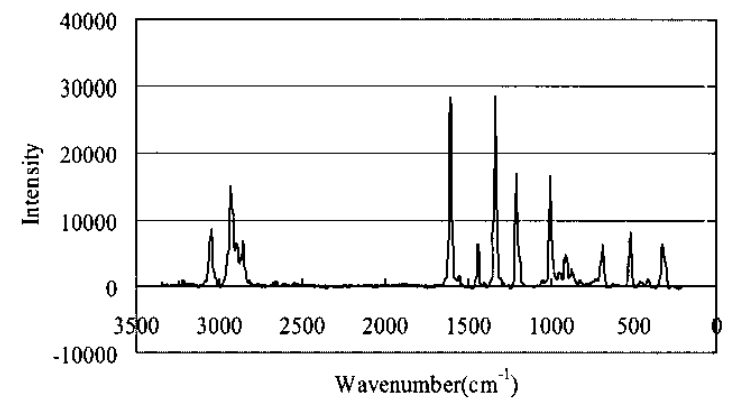

Figure 11: Raman spectrum from non-implanted parylene (Excitation: $514.5 \mathrm{~nm}, 1.0 \mathrm{~mW}$ ). 


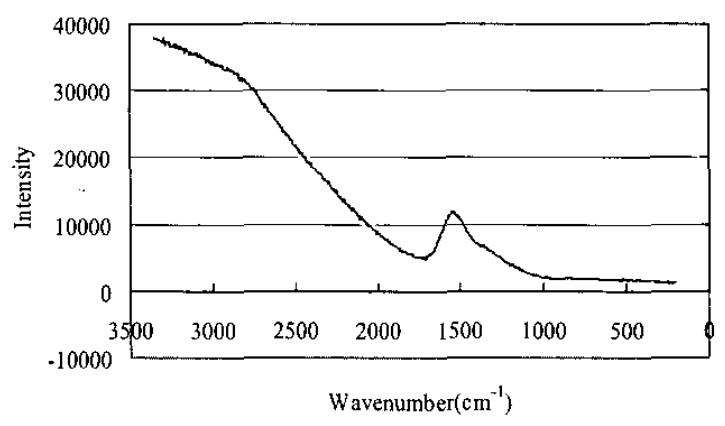

Figure 12: Raman spectrum from non-implanted parylene (Excitation: $514.5 \mathrm{~nm}, 0.1 \mathrm{~mW}$ ).

The sensor chip was tested and calibrated in an isothermal chamber to minimize ambient temperature fluctuations. Compressed nitrogen gas was used to force DI water into the microchannel. The temperature dependence of the sensor's resistance is shown in Figure 13. The resistance (R) decreases linearly with temperature $(\mathrm{T})$, and can be characterized with TCR $(\alpha)$ as follows.

$$
\frac{R(T)}{R\left(T_{0}\right)}=1+\alpha\left(T-T_{0}\right)
$$

The TCR (including the effects of the lead and wire resistance change) was measured to be $-2 \% /{ }^{\circ} \mathrm{C}$, which is an order of magnitude larger than typical metal temperature sensors. This is the main feature of this carbon flow sensor. Figure 14 gives the measured voltage response of the flow sensor when biased at $1.7 \mu \mathrm{A}$ (power consumption of $28 \mu \mathrm{W}$ ). The sensitivity was $380 \mu \mathrm{V} /(\mathrm{nl} / \mathrm{min})$. Figure 15 shows the characteristics of the voltage output in response to the DI water flow. The fluctuation in Figure 15 is believed to be due to the data acquisition system and the ambient temperature fluctuation. These fluctuations can be reduced by compensating the temperature of the entire measurement system.

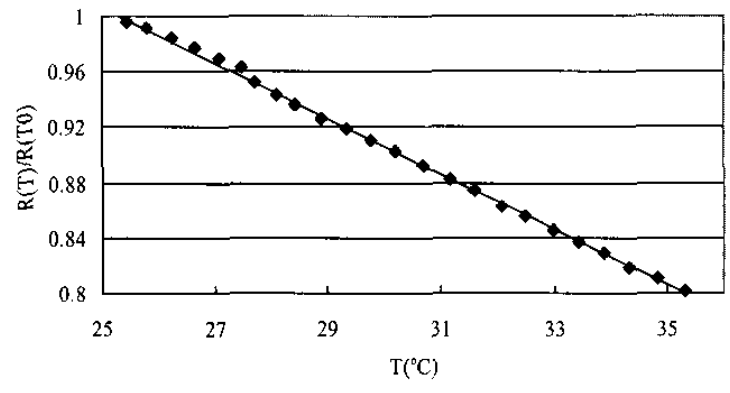

Figure 13: Temperature dependence of resistance. TCR is $-2 \% /{ }^{\circ} \mathrm{C}$.

\section{CONCLUSION}

A nanofluidic carbon flow sensor obtained from ion-implanted parylene has been demonstrated. This sensor

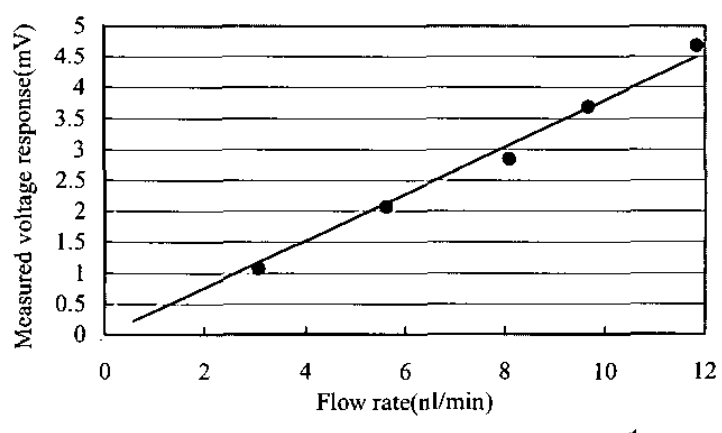

Figure 14: Measured voltage response of the flow sensor that is operated in constant current mode. Bias current is $1.7 \mu$ A. Flow sensitivity is around $380 \mu \mathrm{V} /(\mathrm{nL} / \mathrm{min})$.

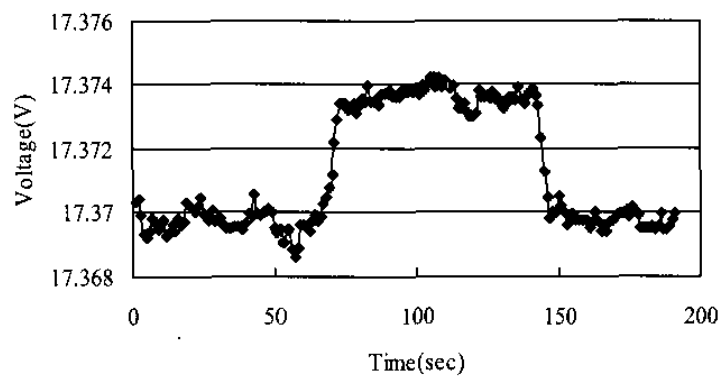

Figure 15: Characteristics of voltage output in response to DI water flow.

can be easily integrated into parylene-based fluidic devices because the sensor is fabricated directly in the parylene by using ion implantation technology. This sensor also enables a fluidic system to be monitored in various ways.

\section{ACKNOWLEDGEMENTS}

The authors would like to thank Trevor Roper for his assistance in the fabrication and Jun Xie and Jason Shih for their helpful discussions.

\section{REFERENCES}

[1] S. Wu, Q. Lin, Y. Yuen, Y.-C. Tai, "MEMS flow sensors for nano-fluidic applications", Sensors and Actuators A: Physical, 89, pp. 152-158, 2001.

[2] M. Ritcher, M. Wackerle, P. Woias, B. Hillerich, "A novel flow sensor with high time resolution based on differential pressure principle", Proceedings of the International Conference on Micro Electro Mechanical Systems(MEMS'99), pp. 118-123, 1999.

[3] J. Xie, J. Shih, Y.-C. Tai, "Integrated surfacemicromachined mass flow controller", Proceedings of the International Conference on Micro Electro Mechanical Systems(MEMS'03), pp. 20-23, 2003.

[4] E. Meng, Ph.D. thesis, Electrical Engineering, California Institute of Technology, 2003. 